\title{
EJERCICIO FÍSICO Y CALIDAD DE VIDA EN ADOLESCENTES SUPERVIVIENTES A UN CÁNCER
}

\section{PHYSICAL EXERCISE AND HEALTH-RELATED QUALITY OF LIFE IN ADOLESCENT CANCER SURVIVORS}

\author{
Carmina Castellano-Tejedor 1,2, Marta Pérez-Campdepadrós ${ }^{2,3}$ y Lluís Capdevila ${ }^{2}$ \\ 1 Departamento de Psiquiatría, Hospital Universitari Vall d'Hebron, CIBERSAM, Universidad Autónoma de \\ Barcelona, España. \\ 2 Departamento de Psicología Básica, Universidad Autónoma de Barcelona, Barcelona, España. \\ ${ }^{3}$ Servicio de Oncología y Hematología Pediátrica. Hospital Universitario Vall d'Hebron, Barcelona, España.
}

Resumen

Objetivo y métodos: La práctica regular de ejercicio físico (EF), es una de las principales conductas relacionadas con un estilo de vida saludable que ha demostrado reportar beneficios tanto físicos como psicológicos sobre la calidad de vida en relación a la salud (CVRS) de las personas. Sin embargo, en España son escasos los trabajos que exploren dicha asociación en población infanto-juvenil superviviente a un cáncer. Por ello, el presente estudio descriptivo mediante encuesta analiza la relación entre el patrón de realización de EF y la CVRS en una muestra de adolescentes oncológicos en remisión (GS), en comparación con un grupo normativo de referencia (GN). Todos los participantes cumplimentaron el cuestionario de salud SF-12 $v_{2}$ y el Autoinforme del Estadio de Cambio para el Ejercicio Físico (AECEF).

Resultados: En ambos grupos había un porcentaje significativamente mayor de varones activos para EF que, además, presentaban puntuaciones significativamente superiores en MCS. No obstante, la CVRS entre ambos grupos era similar e incluso significativamente superior para el GS en MCS. El EF sólo explicó un $12 \%$ de la varianza de MCS en el GN, en conjunción con el género y la edad del superviviente.

Conclusiones: A partir de los resultados, creemos necesario seguir explorando otros fac-
Abstract

Purpose and methods: The regular practice of physical exercise (PE) is one of the main behaviours related with a healthy lifestyle that has demonstrated to yield benefits both on physical and psychological health-related quality of life' domains (HRQoL). However, in Spain, studies exploring such association in pediatric survivors of childhood cancer are scarce. For that reason, this descriptive study examines the relationship between the pattern of PE performance and self-rated $H R Q D L$ in a sample of adolescent survivors of childhood cancer (ASCC), compared to a normative group from the general population (NG). All participants filled in the SF-12 $v_{2}$ questionnaire and the AECEF.

Results: In both groups, a significantly higher percentage of male active survivors was found and they also showed significantly higher mean scores in MCS. However, HRQoL between groups was equivalent and even higher in the case of MCS for the GS. Only $12 \%$ of explained variance for MCS was accounted in the NG, considering $P E$, gender and age at the assessment.

Conclusions: Considering these results, we believe it's needed to explore other factors not addressed in the present work, such as PE intensity or type of physical activity performed that might be mediating the association

\section{Correspondencia:}


tores no contemplados en el presente trabajo, tales como la intensidad o el tipo de actividad física llevada a cabo, que puedan mediar la asociación entre EF y CVRS en supervivientes. También se discute la necesidad de utilizar instrumentos específicos de CVRS para población adolescente superviviente a un cáncer.

Palabras clave: Calidad de vida, ejercicio físico, adolescentes oncológicos en remisión, estudio descriptivo mediante encuesta. between PE and HRQoL in adolescent cancer survivors. It is also discussed the suitability of using specific tools to assess HRQoL in adolescent survivors of childhood cancer populations.

Key words: Health-related quality of life, physical exercise, adolescent survivors of childhood cancer, descriptive study.

emocional $^{(9,10)}$, mejorar el afrontamiento al estrés ${ }^{(11)}$, y disminuir los niveles de ansiedad ${ }^{(12,13)}$ y depresión ${ }^{(14,15)}$. Además, debe destacarse el papel socializador que la práctica regular de EF supone para los adolescentes, sobre todo si se trata de deportes de grupo, como el fútbol, baloncesto, hockey o similares $^{(16)}$. Desgraciadamente, se ha descrito una alta prevalencia de sedentarismo entre la población infanto-juvenil española y europea $^{(17,18)}$ que tenderá a mantenerse hasta la etapa adulta $^{(19)}$, especialmente entre las mujeres $^{(20)}$.

Todo esto cobra especial relevancia si nos centramos en la población de adolescentes oncológicos en remisión. El cáncer infantil representa un $2 \%$ del conjunto de enfermedades neoplásicas con una supervivencia relativa a cinco años tras el diagnóstico cercana al 77\% ${ }^{(21)}$. Esta situación de supervivencia, no obstante, puede ir acompañada de alteraciones físicas a medio-largo plazo, como por ejemplo, cardiotoxicidad, cambios en la composición corporal, alteraciones metabólicas, o fatiga; así como de alteraciones psicológicas, principalmente, distanciamiento social, baja autoestima, ansiedad, depresión y/o estrés ${ }^{(22,23)}$. Estas problemáticas pueden agravarse por la presencia de hábitos poco saludables, como por ejemplo el sedentarismo ${ }^{(24)}$.

Desafortunadamente, se ha observado que un gran porcentaje de los pacientes y supervivientes oncológicos, no son tan acti- 
vos después del tratamiento como lo fueron antes del diagnóstico de cáncer ${ }^{(25-27)}$. Es por ello que la práctica regular de EF se viene proponiendo como una estrategia muy útil y efectiva por su bajo coste socio-sanitario, que podría disminuir o incluso revertir algunos efectos de los tratamientos, favoreciendo así la calidad de vida en relación a la salud (CVRS) del superviviente ${ }^{(28-31)}$.

En España es escasa la literatura científica sobre esta problemática, por lo que sería adecuado confirmar si, tal y como han señalado algunos estudios ${ }^{(25-27,29)}$, los adolescentes oncológicos en remisión son menos activos que los adolescentes de la población general que no han padecido cáncer y si, por otra parte, el EF favorece la CVRS de este colectivo de supervivientes ${ }^{(28-30)}$. Esta información facilitaría el diseño de intervenciones terapéuticas y psicoeducativas en relación a estilos de vida y salud, más ajustadas a las necesidades específicas de esta población.

Por todo ello, este estudio plantea tres objetivos: (1) describir la conducta de realización de EF en una muestra de adolescentes oncológicos en remisión, (2) estudiar y describir su CVRS y (3) analizar la relación entre la práctica de EF y la CVRS. En todos los casos, se compararán los resultados de esta muestra de supervivientes con los obtenidos en una muestra de adolescentes representativos de la población general sin historia previa de cáncer.

\section{MÉTODO}

Se trata de un estudio descriptivo mediante encuesta en el que se ha utilizado un diseño transversal, según la clasificación de Montero y León ${ }^{(32)}$.

\section{Participantes}

Se recogió información de un total de 393 participantes de edades comprendidas entre 14 y 19 años $(M=16,74$, DT=1,32), de los cuales 199 fueron varones (50,6\%) y 194 mujeres $(49,4 \%)$.

El grupo normativo (GN) estaba compuesto por 338 escolares de cuatro centros de educación secundaria obligatoria (E.S.O.) de la ciudad de Barcelona, de los cuales 168 eran varones $(49,7 \%)$ y 170 mujeres (50,3\%). La edad media para este grupo fue de 16,78 años (DT=1,27, rango=14-19). La elección de los participantes del GN se realizó a partir de un muestreo aleatorio estratificado por tipología de centro (públicos y privados).

Por otro lado, el grupo de adolescentes oncológicos en remisión (GS) estaba compuesto por 55 participantes, de los cuales 50 eran residentes en Cataluña (90,9\%) y 5 en otras comunidades autónomas españolas $(9,1 \%)$. Se excluyeron pacientes con tumores del sistema nervioso central y todos aquellos que presentaban comorbilidad psiquiátrica o psicopatológica anterior al diagnóstico oncológico. Para todos ellos, el centro hospitalario de referencia era el Hospital Universitari Vall d'Hebron de Barcelona. En este caso la media de edad fue de 16,53 años (DT=1,57, rango=14-19) y respecto a la distribución por género, contamos con la participación de 31 varones (56,4\%) y 24 mujeres $(43,6 \%)$. Los criterios de inclusión requerían (1) que la edad de los participantes en el momento del diagnóstico estuviese entre 0 y 17 años, ambos incluidos, (2) que la edad en el momento del estudio fuese de entre $14 \mathrm{y}$ 19 años, ambos incluidos, (3) que los participantes fuesen capaces de comprender y hablar castellano o catalán, así como (4) que llevasen un periodo igual o superior a un año fuera de tratamiento oncológico. Todos los participantes fueron voluntarios y firmaron un consentimiento informado.

\section{Instrumentos}

Para la evaluación de la práctica de EF se utilizó el Autoinforme del Estadio de 
Cambio para el Ejercicio Físico (AECEF) de Capdevila ${ }^{(33)}$, basado en el cuestionario de evaluación de la práctica de EF del U.S. Department of Health and Human Services $^{(34)}$. Este autoinforme consta de 8 ítems que permiten obtener información cualitativa (respuesta dicotómica Sí/No) y cuantitativa (frecuencia y duración) acerca de la práctica actual de EF de un individuo. Además, permite situarlo en uno de los cinco estadios de cambio propuestos desde el modelo transteorético de Prochaska ${ }^{(35)}$ : pre-contemplación, contemplación, preparación para la acción, acción y mantenimiento. Estos cinco estadios de cambio pueden agruparse en dos: activos y no activos para el EF. Para la presente investigación se ha considerado activos a los individuos que coinciden con los estadios de acción y mantenimiento respecto al EF; y no activos, a todos aquellos que se encuentren en el resto de estadios (precontemplación, contemplación y preparación para la acción).

Para medir la CVRS se utilizó el cuestionario de salud SF-12 $\mathrm{v}_{2}{ }^{(36)}$ en su adaptación española ${ }^{(37)}$. Este cuestionario está formado por un subconjunto de 12 ítems provenientes del SF-36 ${ }^{(38)}$ cuya información se utiliza para construir las dimensiones de Componente Físico (PCS) y de Componente Mental (MCS) que se valoran en una escala estandarizada en base a normas poblacionales $(M=50, D T=10)$. Los valores superiores o inferiores a 50 deben interpretarse como mejores o peores respectivamente, que los valores de la población de referencia ${ }^{(37)}$. Las normas poblacionales de los componentes sumarios PCS y MCS del SF-12 $v_{2}$, están disponibles para edades a partir de los 18 años; no obstante, tal y como señalan los autores que adaptaron el cuestionario ${ }^{(37-39)}$, éste puede administrarse en edades inferiores siempre y cuando se disponga de un grupo de comparación. El SF-12 $v_{2}$ ha demostrado ser un instrumento adecuado a nuestro ámbito cultural con excelentes propiedades psicométricas ${ }^{(36,40)}$, válido para medir la percepción de salud física y mental de poblaciones muy diversas, así como de fácil y rápida administración (apenas 2 minutos), ya sea en su forma auto-administrada o a través de entrevista semi-estructurada. En nuestro estudio, el Alfa de Cronbach fue de 0,77 (de 0,72 para la dimensión de limitaciones de rol debidas a problemas emocionales a 0,76 para la de función física).

Los datos demográficos (edad, género, estudios y lugar de residencia) se recogieron a través de un protocolo ad hoc desarrollado para la presente investigación.

\section{Procedimiento}

Una vez aprobado el proyecto por el comité de investigación y ética del hospital de referencia, así como por los correspondientes comités directivos de los centros de educación secundaria de la ciudad de Barcelona, se procedió al periodo de reclutamiento y recolección de datos.

Para el GN se llevó a cabo una única sesión de evaluación en cada centro escolar, entre los meses de marzo y mayo del 2007. Tanto el SF- $12 v_{2}$ como el AECEF se cumplimentaron colectivamente de forma auto-administrada en presencia del investigador. En este grupo, la tasa de participación fue del $100 \%$, ya que todos los adolescentes que el día de la evaluación se encontraban en el centro escolar, respondieron a los cuestionarios administrados.

Para obtener los datos del GS, se contactó telefónicamente con todos los pacientes que cumplían criterios de inclusión y que en el momento del estudio tenían programada una visita de control en el Hospital Universitari Vall d'Hebron de Barcelona (entre marzo y mayo del 2008). De los 74 posibles participantes, 7 pacientes $(9,46 \%)$ rechazaron participar 
y $12(16,22 \%)$ no acudieron a la sesión de evaluación a pesar de haber aceptado colaborar en el primer contacto telefónico, por lo que la tasa de participación fue del $74,32 \%$. Para el GS, ambos cuestionarios se cumplimentaron individualmente en un despacho del servicio hospitalario, en forma de entrevista semi-estructurada, siempre con el mismo investigador.

En ambos grupos (GN y GS), el investigador resolvía de forma individual cualquier duda que pudiese surgir en el transcurso de la evaluación.

\section{Análisis estadístico}

El análisis de los resultados se realizó con el programa estadístico SPSS versión 19.0 para Windows. Se verificó la equivalencia en la distribución según el género y la práctica regular de EF (activos vs. no activos) en ambos grupos (GS y GN) con un análisis bivariado de $\chi^{2}$ ( $V$ de Cramer) y según la edad con una prueba t de Student. Las variables género y activos vs. no activos para $E F$, se resumen con su porcentaje válido relativo. Las variables edad, MCS y PCS, se describen con la media, DT y rango. Para la comparación de las puntuaciones MCS y PCS en función del grupo y del género se utilizó la prueba t de Student, acompañándose las medias del intervalo de confianza (IC) del 95\% cuando las diferencias entre grupos resultaron estadísticamente significativas, así como del tamaño del efecto (d de Cohen). Por último, para cada grupo (GN y GS), se llevó a cabo un análisis de regresión lineal (pasos sucesivos) para estimar el porcentaje de varianza explicada de MCS y PCS en función de los factores explicativos contemplados en la presente investigación (género, edad y práctica de EF). La significación de todas las pruebas se ha considerado a un nivel de probabilidad del 5\% o inferior, indicando siempre la significación exacta que ofrece el paquete estadístico SPSS.

\section{RESULTADOS}

No hubo diferencias significativas entre grupos en cuanto a la distribución por edad (GN: $M=16,78, \mathrm{DT}=1,27$, rango=14-19; GS: $M=16,53, \mathrm{DT}=1,57$, rango=14-19) o género $(\mathrm{GN}=49,7 \%$ varones y $50,3 \%$ mujeres; $G S=56,4 \%$ varones y $43,6 \%$ mujeres), por lo que puede considerarse que ambos grupos eran equivalentes respecto a esas variables.

Resultados en relación al objetivo (1): describir la conducta de realización de EF en el GS en comparación con el GN

No se observaron diferencias estadísticamente significativas entre grupos en cuanto al porcentaje de individuos activos para el $E F(\mathrm{GN}=65,1 \%$ adolescentes activos; $\mathrm{GS}=56,4 \%$ adolescentes activos). Sin embargo, tanto en el GN como en el GS, se observaron diferencias intragrupo estadísticamente significativas en relación al género y la practica regular de EF. Así, en el GN había un 64,1\% de varones activos frente a un $35,9 \%$ de mujeres activas $\left(\chi_{(1, N=338)}^{2}=52,17 ; p<0,001\right.$; $\mathrm{V}=0,4)$. Lo mismo sucedía en el caso del GS $\left(\chi_{(1, N=55)}^{2}=3,74 ; p=0,048 ; V=0,3\right)$, siendo de nuevo superior el porcentaje de varones activos $(67,7 \%)$ en comparación con el de mujeres $(32,3 \%)$.

Resultados en relación al objetivo (2): estudiar y describir la CVRS en el GS en comparación con el GN

La Tabla 1 refleja las puntuaciones de CVRS en cada una de las dos dimensiones del SF-12 $v_{2}$ para cada grupo.

Ambos grupos eran equivalentes en la dimensión PCS; sin embargo, para la dimensión MCS el GS mostró una puntuación significativamente superior al GN $\left(\mathrm{t}_{391}=-3,869, \mathrm{p}<0,000 ; \mathrm{d}=0,59\right.$, IC $(95 \%)$ $-7,65$ a $-2,49)$. Por otro lado, tal y como 
Tabla 1. Puntuaciones de MCS y PCS del SF-12

\begin{tabular}{|l|c|c|c|}
\cline { 2 - 4 } \multicolumn{1}{c|}{} & Grupo Normativo $(\mathrm{n}=338)$ & Supervivientes $(\mathrm{n}=55)$ & \\
\cline { 2 - 4 } \multicolumn{1}{c|}{} & Media (DT) & Media (DT) & $\mathbf{p}$ \\
\hline MCS & $47,85(9,17)$ & $52,93(8,03)$ & $<0,000^{*}$ \\
\hline PCS & $52,72(6,15)$ & $54,67(7,33)$ & n.s. \\
\hline
\end{tabular}

MCS: Componente mental; PCS: Componente físico.

n.s.= diferencias no significativas estadísticamente.

(*) Significación estadística según la prueba t de Student.

Tabla 2. Puntuaciones de MCS y PCS del SF-12 2 por grupo y género

\begin{tabular}{|c|c|c|c|c|c|c|c|}
\hline & \multirow[b]{2}{*}{ Género } & \multicolumn{3}{|c|}{ Grupo Normativo $(n=338)$} & \multicolumn{3}{|c|}{ Supervivientes $(n=55)$} \\
\hline & & Media (DT) & Rango & $\mathbf{p}$ & Media (DT) & Rango & p \\
\hline \multirow{2}{*}{ MCS } & Hombres & $50,51(7,96)$ & $25,58-66,61$ & \multirow{2}{*}{$<0,000^{*}$} & $54,84(7,06)$ & $39,33-64,94$ & \multirow{2}{*}{$0,043^{*}$} \\
\hline & Mujeres & $45,23(9,54)$ & $12,97-68,27$ & & $50,45(8,68)$ & $24,22-62,49$ & \\
\hline \multirow{2}{*}{ PCS } & Hombres & $52,30(6,44)$ & $22,38-63,11$ & \multirow{2}{*}{ n.s. } & $53,78(8,31)$ & $27,88-64,11$ & \multirow{2}{*}{ n.s. } \\
\hline & Mujeres & $53,14(5,83)$ & $33,78-66,12$ & & $55,82(5,79)$ & $37,69-62,33$ & \\
\hline
\end{tabular}

MCS: Componente mental; PCS: Componente físico.

n.s.= diferencias no significativas estadísticamente.

(*) Significación estadística según la prueba t de Student.

puede observarse en la Tabla 2, dentro del GS no se encontraron diferencias entre géneros para los valores de PCS pero sí para los de MCS $\left(t_{53}=2,070, p=0,043 ; d=0,55\right.$, IC (95\%) 0,14 a 8,64). Lo mismo sucedía en el GN, dónde las mujeres mostraban puntuaciones promedio significativamente inferiores en bienestar psicológico (MCS) en comparación con los varones $\left(\mathrm{t}_{336}=5,523\right.$, $p<0,000 ; d=0,60$, IC (95\%) 3,40 a 7,16$)$.

Resultados en relación al objetivo (3): analizar la relación entre la práctica de EF y la CVRS en el GS y comparar con el GN

En el GN, MCS y práctica de EF (min/ sem) correlacionaron significativamente $(r=0,244, p<0,000)$. Así, los adolescentes activos para $E F(M=49,60, \mathrm{DT}=8,50$, rango=19,49-66,41) puntuaban significativamente más alto que los no activos ( $M=44,59$, $\mathrm{DT}=9,50$, rango $=12,97-68,27)$ en el $\mathrm{MCS}$ del SF-12 $2_{\mathrm{v} 2}\left(\mathrm{t}_{336}=-4,956, \mathrm{p}<0,000, \mathrm{~d}=0,26\right.$, IC $95 \%-7,00$ a $-3,02)$. Sin embargo, en el GS la práctica de EF ( $\mathrm{min} / \mathrm{sem})$ no correlacionó con ninguna de las puntuaciones de CVRS (ni MCS, ni PCS).

Por último, para analizar la relación entre los distintos factores explicativos propuestos en el presente estudio ( $E F$, género y edad) y las dos dimensiones de CVRS estudiadas (MCS y PCS), se plantearon dos análisis de regresión (por pasos sucesivos) para cada grupo. 
$\mathrm{Ni}$ en el GN ni en el GS se obtuvieron modelos significativos para PCS con ninguna de las variables consideradas. Lo mismo sucedió para MCS en el caso del GS. En cambio, en el GN se obtuvo un modelo de regresión para MCS $\left(\mathrm{R}^{2}\right.$ corregida $=12 \%$, $F=16,079, \quad g l=337, p<0,000)$ que a partir de las variables $E F(\beta=, 162, p=0,004$, IC $95 \% 1,008-5,226)$, género $(\beta=-0,223$, $\mathrm{p}<, 000$, IC $95 \%-6,088--2,080)$ y la edad del adolescente $(\beta=-0,132, \quad p=0,011, \quad I C$ $95 \%-1.675--0,220)$, explicaba un $12 \%$ de la varianza de MCS.

\section{DISCUSIÓN Y CONCLUSIONES}

La presente investigación tenía tres objetivos: (1) estudiar y describir la práctica de EF en una muestra de adolescentes supervivientes a un cáncer (GS), (2) estudiar y describir su CVRS y (3) explorar la posible asociación entre EF y CVRS. Todos los resultados del GS se comparan con los obtenidos en el GN.

Al analizar las pautas de EF en ambas muestras, observamos que, contrariamente a lo que sugieren algunos estudios ${ }^{(26,27)}$, los adolescentes oncológicos en remisión practican EF con una frecuencia similar a la que lo hacen sus iguales de la población general. Asimismo, es preciso destacar que en ambos grupos (GN y GS) encontramos un porcentaje significativamente superior de varones activos para el EF que de mujeres. Otros autores habían señalado resultados similares ${ }^{(20,30)}$. Considerando estos resultados, podemos inferir que los adolescentes del GS presentan un patrón de realización de EF similar al de los adolescentes de la población general sin historia previa de cáncer.

Por otra parte, también hemos podido observar como el GS manifiesta una CVRS satisfactoria $y$, también en este caso, similar a la que se observa en su grupo de referencia sin antecedentes oncológicos. Es más, el GS ha mostrado una percepción de bienestar psicológico (MCS) significativamente superior a la del GN. Esto podría explicarse porque haber sobrevivido a un cáncer puede Ilevar a estos adolescentes a valorar más su estado de salud actual o incluso, en cierta medida, a sobrestimarlo ${ }^{(41,42)}$. Otra posible explicación es que hayan experimentado cierta maduración personal o crecimiento postraumático que podría estar vinculado a una mayor percepción de bienestar psicológico, tal y como han señalado algunos autores ${ }^{(43-46)}$.

Contrariamente a lo esperado ${ }^{(47)}$, sólo se ha observado una relación estadísticamente significativa entre práctica de EF y CVRS. Este ha sido el caso de la práctica regular de EF en el GN y su mayor percepción de bienestar psicológico (MCS). En el caso del GS, el EF no ha mostrado relación con ninguna de las dos dimensiones de CVRS valoradas en el presente trabajo. Así, a pesar de los múltiples beneficios del EF sobre la CVRS de los individuos descritos en la literatura científica ${ }^{(1-16)}$, esta relación no ha podido observarse en nuestra muestra. No obstante, vale la pena indicar que sí hemos observado una tendencia a presentar mayores puntuaciones promedio de CVRS entre los adolescentes activos para EF, tanto en el GN como en el GS. Así, este dato no debe pasarse por alto por su relevancia a nivel clínico ${ }^{(1,16,29)}$, a pesar de que no alcance criterios de significación estadística. En referencia a este último aspecto, pensamos que esta ausencia de relación podría ser debida a diversas causas.

En primer lugar, el tipo de evaluación de la práctica de EF. A pesar de tratarse de un instrumento adaptado a nuestro propio entorno cultural y de presentar adecuadas propiedades psicométricas ${ }^{(33)}$, el AECEF es un instrumento que se limita a valorar la frecuencia y duración de la práctica de EF y, en consecuencia, a clasificar a los individuos en diferentes estadios de cam- 
bio; pero no recoge ni la intensidad de dicho EF (p.e. baja, moderada, intensa), ni el tipo de actividad concreta llevada a cabo. Así, tales aspectos podrían ser determinantes para poder ahondar en el tipo de relación existente entre EF y CVRS. Aunque son escasos los trabajos que han valorado tales aspectos en población oncológica superviviente a un cáncer, un estudio de revisión reciente ${ }^{(48)}$ identificó 17 estudios en este ámbito. Los resultados de dicha revisión confirmaron que las intervenciones clínicas basadas en el EF para población adolescente con cáncer, son factibles y seguras, en especial para las leucemias linfoblásticas agudas pacientes y durante el tratamiento médico. En ningún trabajo se reportaron efectos adversos y sí efectos beneficiosos sobre la fatiga, la fuerza y la CVRS. Además, algunos trabajos señalaban efectos positivos sobre el sistema inmunológico, la composición corporal, el sueño, los niveles de actividad, diversos aspectos de la función física e incluso, aspectos específicos de la adolescencia como la reintegración en el grupo de iguales a través del EF. En resumen, la evidencia de las intervenciones de EF en oncología pediátrica está clasificada en un nivel "3" (48). No obstante, y a pesar de lo prometedores que son esos resultados, se necesitan más trabajos de investigación con gran rigor metodológico y que consideren los aspectos específicos de los supervivientes de cáncer en edad adolescente, para poder establecer recomendaciones de EF basadas en la evidencia.

Un segundo aspecto que podría estar influyendo en la incapacidad para observar relación entre el EF y la CVRS en el GS de nuestro trabajo, es la elección de un cuestionario genérico para evaluar CVRS. A pesar de que el SF-12 12 es un instrumento válido y fiable ${ }^{(36)}$ y de fácil y rápida administración ${ }^{(37)}$, éste podría no ser capaz de recoger algunos aspectos re- levantes de la CVRS de los adolescentes oncológicos en remisión que podrían verse beneficiados con una práctica regular de EF. Probablemente, eso explicaría el que sólo hayamos encontrado una relación estadísticamente significativa entre EF y MCS en el GN. Por otro lado, no deja de ser sorprendente que no se haya repetido dicha asociación con el PCS. Algunos autores ${ }^{(49,50)}$ ya han señalado la necesidad de desarrollar instrumentos más "finos" capaces de recoger todos aquellos aspectos relevantes de una población tan particular como son los adolescentes oncológicos en remisión. Aunque todavía no disponemos de cuestionarios adaptados a nuestro propio entorno cultural para población adolescente, ya existen algunos instrumentos de valoración del impacto de cáncer para supervivientes en etapa pediátrica $^{(49-51)}$.

En resumen, son necesarios más trabajos que permitan contrastar los resultados obtenidos y que aporten mayor claridad acerca del papel que juega la práctica regular de EF en la CVRS de la población de adolescentes oncológicos en remisión.

De cara a futuras investigaciones creemos interesante replicar este trabajo con una muestra clínica de mayor tamaño y a poder ser, con diseños longitudinales en los que, además, se controle el tipo de secuelas que esta población pueda presentar. Es importante resaltar que el diseño longitudinal nos permitiría controlar el efecto del tiempo transcurrido desde la finalización del tratamiento sobre la salud percibida, ya que algunos estudios parecen indicar que la percepción de la misma, presenta un declive más rápido que el que se da en la población general, sobre todo, en lo que a salud física se refiere ${ }^{(41)}$. Como ya hemos comentado previamente, los estudios empíricos con población pediátrica en nuestro país son escasos, y más aún en el ámbito específico de la psico-oncología; por todo ello, es 
necesaria más investigación acerca de la CVRS y de las variables favorecedoras de la misma en el colectivo de supervivientes.

Conflictos de interés: Los autores del presente trabajo declaran no tener ningún conflicto de interés.

\section{Agradecimientos}

Esta investigación se realizó gracias a la colaboración del Hospital Universitari Vall d'Hebron de Barcelona (España), de los institutos I.E.S. Fort Pius, I.E.S. Montseny, I.E.S. Miquel Tarradell y el Colegio Sant Estanislau de Kostka, todos ellos de Barcelona (España), así como de las ayudas FPI 00286 de la Universitat Autònoma de Barcelona y PSI2011-29807-C03-01/PSIC del Ministerio de Ciencia e Innovación. Finalmente, agradecemos a todos los participantes del estudio su colaboración desinteresada en el mismo.

\section{REFERENCIAS BIBLIOGRÁFICAS}

1. American College of Sports Medicine [ACSM]. Physical Activity Guidelines for Americans. 2008. [Acceso el 17 de diciembre de 2008]. Disponible en: http://www. health.gov/paguidelines/pdf/paguide.pdf.

2. Escolar JL, Pérez-Romero de la Cruz C, Corrales R. Actividad física y enfermedad. An Med Interna 2003;20:427-33.

3. Jiménez MG, Martínez P, Miró E, Sánchez Al. Bienestar psicológico y hábitos saludables: ¿están asociados a la práctica de ejercicio físico? Int J Clin Health Psychol 2008;8:185-202.

4. Boreham C, Twisk J, Neville C, Savage M, Murray L, Gallagher A. Associations between physical activity patterns during adolescence and cardiovascular risk factors in young adulthood: The Northern Ireland Young Hearts Project. Int J Sport Med 2002;23(Suppl. 1):S22-26.
5. Lawlor DA, Leon DA. Association of Body Mass Index and Obesity measured in early childhood with risk of coronary heart disease and stroke in middle age: findings from the Aberdeen Children of the 1950s prospective cohort study. Circulation 2005;111;1891-6. Doi:10.1161/01. CIR.0000161798.45728.4D

6. Lefevre J, Philippaerts R, Delvaux K, Thomis M, Claessens A, Lysens R. et al. Relationship between cardiovascular risk factors at adult age, and physical activity during youth and adulthood. Int J Sports Med 2002;23 (Suppl. 1),S32-38.

7. Rowland TW. Promoting physical activity for children's health: rational and strategies. Sports Med 2007;37:929-36.

8. Ekeland E, Heian F, Hagen KB. Can exercise improve self-esteem in children and young people? A systematic review of randomised controlled trials. BR J Sports Med 2005;39:792-8. Doi:10.1136/ bjsm.2004.017707

9. Biddle SJ, Gorely T, Stensel DJ. Healthenhancing physical activity and sedentary behaviour in children and adolescents. J Sports Sci 2004;22:679-701. Doi 10.1080/02640410410001712412:

10. Sagatun A, Sogaard AJ, Bjertness E, Selmer $\mathrm{R}$, Heyerdahl S. The association between weekly hours of physical activity and mental health: A three-year follow-up study of 15-16-year-old students in the city of Oslo, Norway. BMC Public Health 2007;7:15563. Doi: 10.1186/1471-2458-7-155

11. Everly GS, Lating JM. Physical exercise and the human stress response. En Everly GS, Lating JM, editors. A clinical guide to the treatment of the human stress response $\left(2^{\text {nd }}\right.$ Ed.). Kluwer Academic Publishers: New York, 2002.p.269-285

12. Akandere $M$, Tekin A. Efectos del Ejercicio Físico sobre la ansiedad. En línea. 2005. [Acceso el 12 de Noviembre de 2008] Disponible en: http://www. sobreentrenamiento.com/publice/Articulo. asp? ida $=478$ 
13. Gutiérrez-Calvo M, Espino O, Palenzuela DL, Jiménez A. Ejercicio físico regular y reducción de la ansiedad en jóvenes. Psicothema 1997;9 :499-508.

14. Lawlor DA, Hopker SW. The effectiveness of exercise as an intervention in the management of depression: Systematic review and meta-regression analysis of randomised controlled trials. BMJ 2001;322:763-7. Doi: 10.1136/ bmj.322.7289.763

15. Palenzuela DL, Gutiérrez-Calvo MG, Avero P. Ejercicio físico regular como un mecanismo de protección contra la depresión en jóvenes. Psicothema 1998;10: 29-39.

16. Bailey R. Physical education and sport in schools: a review of benefits and outcomes. J Sch Health 2006;6:397-401. Doi:10.1111/j.1746-1561.2006.00132.x

17. Roman B, Serra-Majem L, Ribas-Barba L, Pérez-Rodrigo C, Aranceta J. How many children and adolescents in Spain comply with the recommendations on physical activity? J Sports Med Phys Fitness 2008; 8:380-7.

18. Sallis J. Age-related decline in physical activity: A synthesis of human and animal studies. Med SciSports Exerc 2000;32:1598600. Doi:10.1097/00005768-20000900000012

19. Lefevre J, Philippaerts R, Delvaux K, Thomis M, Vanreusel B, Vanden Eynde B, et al. Daily physical activity and physical fitness from adolescence to adulthood: A longitudinal study. AM J Hum Biol 2000;12:487-97. Doi:10.1002/15206300(200007/08)12:4\%3C487::AIDAJHB8\%3E3.3.CO;2-N

20. De Bourdeaudhuij L, Sallis J, Vandelanotte C. Tracking and explanation of physical activity from adolescence to young adulthood. A 7-year prospective study. Res Q Exerc Sport 2002;73:376-85. Doi:10.1186/1479-5868-1-14

21. Peris-Bonet R, García A, Muñoz F, Pardo E, Comisión científica de colaboradores de la SEOP. Registro Nacional de Tumores Infantiles (Informe estadístico RNTI-SEOP). Estadísticas básicas 1980-2006. Valencia: Universidad de Valencia, 2007.

22. Heath JA. Monitoring after childhood cancer. Aus Fam Physician 2005;34: 7617.

23. Ness K, Gurney J. Adverse late effects of childhood cancer and its treatment on health and performance. Ann Rev Public Health 2007;28:279-302. Doi:10.1146/ annurev.publhealth.28.021406.144049

24. Hudson MM, Mertens AC, Yasui Y, Hobbie W, Chen H, Gurney JG, et al. Health status of adult long-term survivors of childhood cancer: A report from the Childhood Cancer Survivor Study. JAMA 2003;290:1583-92. Doi:10.1001/jama.290.12.1583

25. Clarke S, Eiser C. Health behaviours in childhood cancer survivors: A systematic review. Eur J Cancer 2007;43:1373-84. Doi: 10.1016/j.ejca.2007.03.002

26. Keats MR, Culos-Reed NS, Courneya KS, McBride M. J Pediatr Oncol Nurs 2006; 23: 135-42. Doi: 10.1177/1043454206287304

27. Reeves M, Eakin E, Lawler S, Wahnefried WD. Health Behaviours in survivors of childhood cancer. Aus Fam Physician 2007;36:95-6.

28. Braith RW. Role of exercise in rehabilitation of cancer survivors. Pediatr Blood Cancer 2005;44:595-9. Doi: 10.1002/pbc.20354

29. Children's Oncology Group. Health Link. Healthy living after treatment for childhood cancer. Cure search. En línea. 2006. [Acceso el 17 de diciembre de 2008]. Disponible: http://www. survivorshipguidelines.org

30. Schmitz KH, Holtzman J, Courneya KS, Mâsse LC, Duval S, Kane R. Controlled physical activity trials in cancer Survivors: A systematic review and meta-analysis. Cancer Epidemiol Biomarkers Prev 2005;14:1588-95. Doi: 10.1158/10559965.EPI-04-0703

31. Van Weert E, Hoekstra-Weebers J, Grol B, Otter R, Arendzen HJ, Postema K, et al. 
A multidimensional cancer rehabilitation program for cancer survivors. Effectiveness on health related quality of life. J Psychosom Res 2005;58:485-96. Doi: 10.1016/j.jpsychores.2005.02.008

32. Montero I, León OG. A guide for naming research studies in Psychology. Int J Clin Health Psychol 2007;7:847-62.

33. Capdevila L. Actividad física y estilo de vida saludable ( $1^{\text {a }}$ Edición). Girona: Documenta Universitaria, 2005.

34. U.S. Department of Health and Human Services [USDHHS]. Promoting physical activity. A guide for community action. Champaign, I.L.: Human Kinetics, 1999.

35. Prochaska JO, Marcus BH. The transtheoretical model: Applications to exercise. En: Dishman RK, editor. Advances in exercise adherence. Champaign, I.L.: Human Kinetics, 1994.p.161-80

36. Ware JE Jr, Kosinski M, Keller SD. A 12-Item Short Form Health Survey: construction of scales and preliminary tests of reliability and validity. Med Care 1996;34:220-33. Doi:10.1097/00005650-19960300000003

37. Institut Municipal d'Investigació Mèdica [IMIM-IMAS] (2002). Puntuación del instrumento. Cuestionario de Salud SF-12 versión 2. Versión española del SF-36 $v_{2}$, Health Survey 1994, 2002, adaptada por J. Alonso et al. (Puntuación_SF - 12 $\mathrm{v}_{2-}$ BiblioPRO.pdf). [Acceso el 18 de enero de 2008] Disponible: http://iryss.imim.es/ iryss/new-ficha.asp?id=243.

38. Vilagut G, Ferrer M, Rajmil L, Rebollo P, Permanyer-Miralda G, Quintana JM, et al. El Cuestionario de Salud SF-36 español: una década de experiencia y nuevos desarrollos. Gac Sanit 2005;19:135-50.

39. Rebollo P. Utilización del "grupo SF" de los cuestionarios de calidad de vida (SF36, SF-12 y SF-6D) en el marco de ensayos clínicos y en la práctica clínica habitual. Med Clin (Barc) 2008;130:740-1.

40. Gandek B, Ware JE, Aaronson NK, Apolone G, Bjorner JB, Brazier JE, et al.
Cross-validation of item selection and scoring for the SF-12 Health Survey in nine countries: results from the IQOLA Project. International Quality of Life Assessment. J Clin Epidemiol 1998;51:1171-8

41. Reulen RC, Winter DL, Lancashire ER, Zeegers MP, Jenney ME, Walters SJ, et al. Health-status of adult survivors of childhood cancer: a large-scale population-based study from the British Childhood Cancer Survivor Study. Int J Cancer 2007;121:63340. Doi:10.1002/ijc.22658

42. Castellano C, Blasco T, Oller A, PérezCampdepadrós M, Sánchez de Toledo J, Capdevila L. [Quality of life in adolescent survivors of childhood cancer]. Med Clin (Barc) 2009;133:783-6.

43. Eiser C, Hill JJ, Vance YH. Examining the psychological consequences of surviving childhood cancer: Systematic review as a research method in pediatric psychology. J Pediatr Psychol 2000;25:449-60. Doi: 10.1093/jpepsy/25.6.449

44. Joseph S, Linley P. Growth following adversity: Theoretical perspectives and implications for clinical practice. Clin Psychol Rev 2006;26:1041-53. Doi: 10.1016/j.cpr.2005.12.006

45. Mattsson E, Ringnér A, Ljungman G, von Essen L. Positive and negative consequences with regard to cancer during adolescence. Experiences two years after diagnosis. Psychooncology 2007;16:1003-9. Doi: 10.1002/pon.1162

46. Sumalla EC, Ochoa C, Blanco I. Posttraumatic growth in cancer: Reality or illusion? Clin Psychol Rev 2009;29:24-33. Doi: 0.1016/j.cpr.2008.09.006

47. Castellano C, Pérez-Campdepadrós $M$, Capdevila L, Sánchez de Toledo J, Gallego S, Blasco T. Surviving childhood cancer: Relationship between exercise and coping on quality of life. Span J Psychol 2013;16(E1) Doi:10.1017/sjp.2013.1

48. Baumann FT, Bloch W, Beulertz J. Clinical exercise interventions in pediatric oncology: A systematic review. Pediatr 
Res 2013;74: 366-374. Doi: 10.1038/ pr.2013.123

49. Zebrack BJ, Landier W. The perceived impact of cancer on quality of life for posttreatment survivors of childhood cancer. Qual Life Res 2011;20:1595-608.

50. Zebrack B. Developing a new instrument to assess the impact of cancer in young adult survivors of childhood cancer.
J Cancer Surviv 2009;3:174-80. Doi: 10.1007/s11764-009-0087-0

51. Zebrack BJ, Donohue JE, Gurney JG, Chesler MA, Bhatia S, Landier W. Psychometric evaluation of the impact of Cancer (IOC-CS) scale for young adult survivors of childhood cancer. Qual Life Res 2010;19:207-18. Doi:10.1007/ s11136-009-9576-x 